\title{
Industrie 4.0 - Chance für Europas Wirtschaft
}

\author{
R. Ploss
}

Online publiziert am 19. September 2014

(c) Springer Verlag Wien 2014

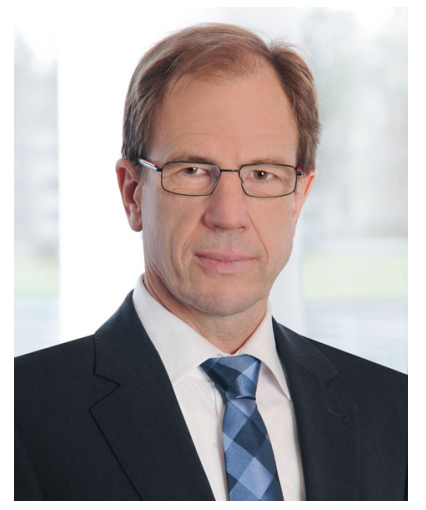

Dr. Reinhard Ploss
Die Fabrik wird intelligent durch die Verbindung von Elektronik, Mechanik und übergreifenden Systemansätzen. Die Mikroelektronik ist der entscheidende Faktor, sie steuert mit ihren Chips Abläufe und schützt Daten. Intelligente Maschinen, Lagersysteme und Betriebsmittel tauschen untereinander Informationen aus, lösen auf dieser Grundlage Aktivitäten aus und steuern und optimieren sich gegenseitig. Die Fabriken

und Lager von Lieferanten und Kunden werden ebenfalls vernetzt. Und die reibungslose Aufnahme von innovativen Produkten in der Fertigung, an denen Entwickler ständig arbeiten, wird in der Regel bei bestehendem Betrieb geschafft.

Halbleiter spielen dabei eine wichtige Rolle. Hardwarebasierte Authentifizierungssysteme bieten einen wirkungsvollen Schutz vor Manipulationen und Sabotage. Mikrocontroller, Leistungselektronik und Sensoren ermöglichen eine immer stärkere Automatisierung Die Energieeffizienz steigt durch die bessere Steuerung elektrischer Motoren - dank Halbleitern. Die europäische Wirtschaft hat die einmalige Chance, dieses Zukunftsprojekt voranzutreiben. Dies wäre auch eine wichtige Säule für die dringend notwendige Reindustrialisierung in der EU. Dafür gilt es, Europas Stärken zu nutzen, beispielsweise die gewachsene Vernetzung in der Wertschöpfungskette. Aber auch Daten- und Kommunikationssicherheit, Erfahrungen bei der Automatisierung und Standardisierung können zu Wettbewerbsvorteilen werden.

Infineon hat schon heute einige Elemente des Konzepts Industrie 4.0 umgesetzt. Die Fertigung von Halbleitern gehört zu den komplexesten Produktionssystemen der Welt. Millionen von einzelnen
Elementen auf den Chips müssen funktionieren. Wir können einzelne Chips über die Wertschöpfungskette präzise nachverfolgen. So verknüpfen wir in Echtzeit Testergebnisse aus unseren Werken in Asien etwa mit den Produktionsschritten unserer Fertigung in Dresden. Wir können nicht nur gezielt optimieren, sondern wir lernen auch schneller. Für innovative Technologien und Produkte ist das ein unschätzbarer Vorteil. Diesen Weg setzen wir konsequent fort. In Villach entsteht dafür der "Pilotraum Industrie 4.0": eine hochautomatisierte Fertigung auf Grundlage eines cyber-physischen Systems, in dem die Interaktion von Mensch und Maschine eine neue Dimension erreichen wird.

Soll die Industrie in Europa und darüber hinaus in Zukunft über die gesamte Wertschöpfungskette integriert werden, brauchen wir vor allem eines: Sicherheit beim Datenaustausch und Schutz der vernetzten Systeme gegen Hacker-Angriffe. Produkteigenschaften, Baupläne, Fertigungsabläufe sind wettbewerbsrelevante und sensible Informationen: Diese Daten und die Produktion müssen vor Angriffen geschützt, der Zugang zu Informationen klar reglementiert werden. Fortschrittliche Sicherheitstechnik - bestehend aus einem geschlossenen System von zertifizierter Hard- und Software - kann diese Probleme lösen.

Die Politik hat die wichtige Aufgabe, sowohl technologische als auch rechtliche Standards festzuschreiben. Industrie 4.0 setzt auf Systeme, die über das Internet vernetzt sind. Dazu gibt es keine Alternative. Wie verletzlich diese Infrastruktur ist, haben die jüngsten Diskussionen gezeigt. Politik und Wissenschaft können zudem wichtige Brückenbauer zu und zwischen den Unternehmen sein. Zum Beispiel, indem sie das Bewusstsein für erhöhte Sicherheit fördern und den Aufbau von vertrauenswürdigen Kommunikationskonzepten unterstützen. Forschungsprojekte und Kooperationen müssen zudem transparent durchgeführt werden. Sie dienen als Plattform, um auch die wettbewerbsrechtlichen Anforderungen zu erfüllen.

Schaffen wir den Schulterschluss, dann wird die europäische Industrie der erfolgreiche Treiber der nächsten industriellen Revolution - als Produktionsstandort und als Fabrikausrüster der Welt. 